Supporting Information

\title{
Interfacial Triggering of Conductive Filament Growth in Organic Flexible Memristor for High Reliability and Uniformity
}

Sin-Hyung Lee ${ }^{\dagger}$, Hea-Lim Park', Min-Hoi Kim", Sujie Kang ${ }^{\dagger}$, and Sin-Doo Lee ${ }^{\star}, t$

'School of Electrical Engineering, 1 Gwanak-ro, Gwanak-ku, Seoul National University,

Seoul 08826, Republic of Korea

"Department of Creative Convergence Engineering, Hanbat National University,

Yuseong-ku, Daejeon 305-719, Republic of Korea

Corresponding Author

*E-mail: lclab2@snu.ac.kr (S.-D. Lee) 
SUPPLEMENTARY FIGURES

(a)

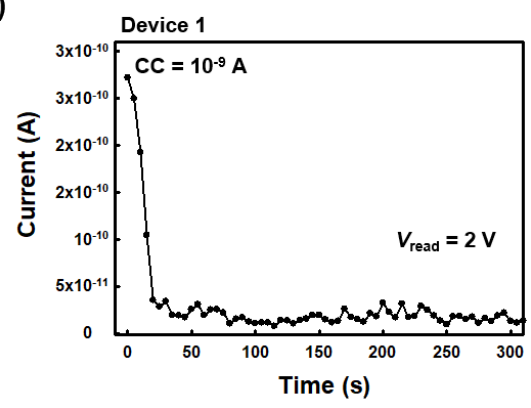

(b)

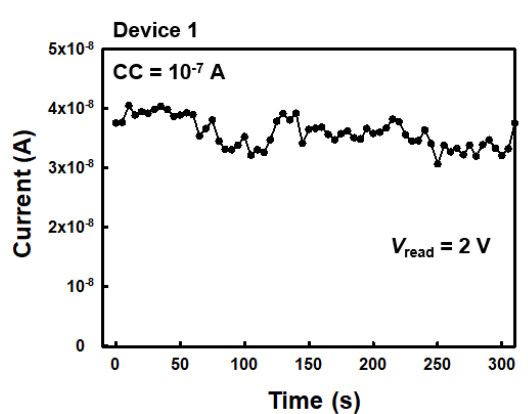

Figure S1. The effect of the compliance current (CC) on the electrical characteristics of the organic ECM device. Memory retention tests of Device 1 in the low resistance state at $\underline{V}_{\text {read }}=2 \mathrm{~V}$. Two different values of the external $\mathrm{CC}$ were used in the writing process; (a) $10^{-9} \mathrm{~A}$ and (b) $10^{-7} \mathrm{~A}$.

(a)

(b)
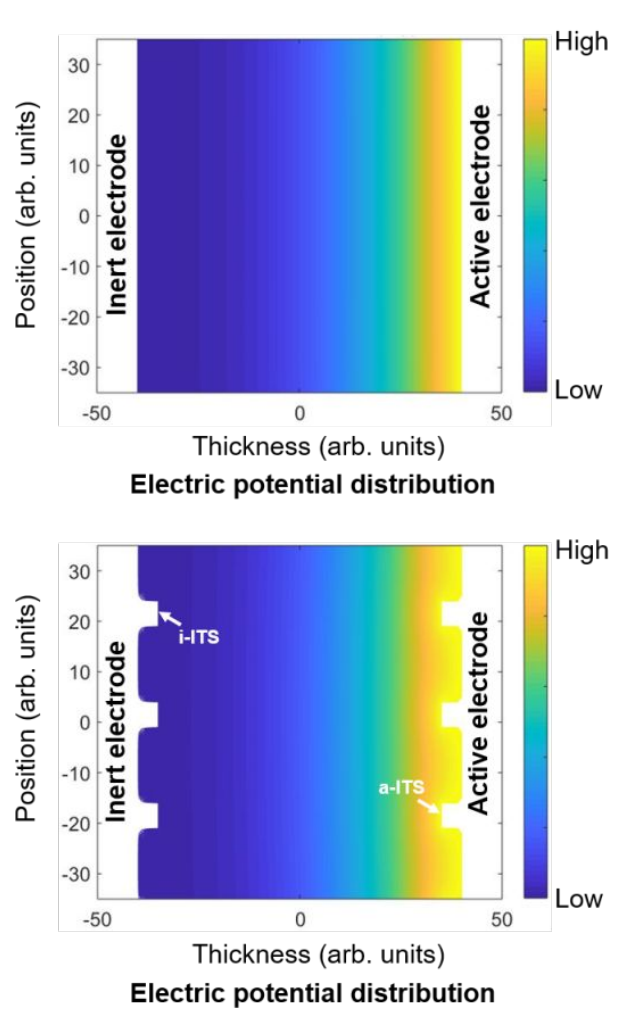
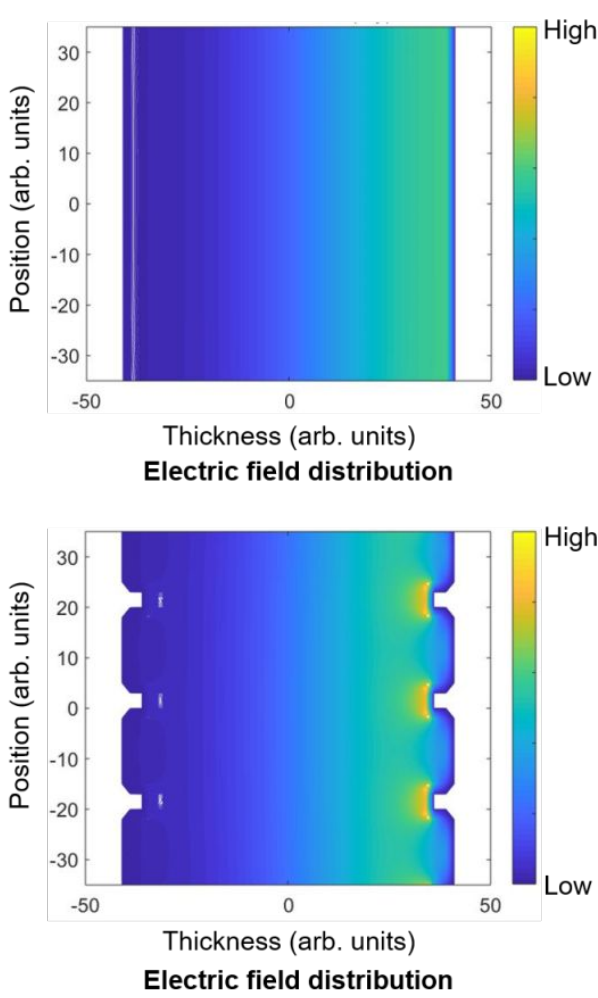

Figure S2. The simulation results for the distributions of the electric potential and the electric field in (a) the device without an interfacial triggering site (ITS) and (b) the device with ITSs. Based on a 2-dimensional finite difference method, the Poisson equation was solved using MATLAB. 
(a)

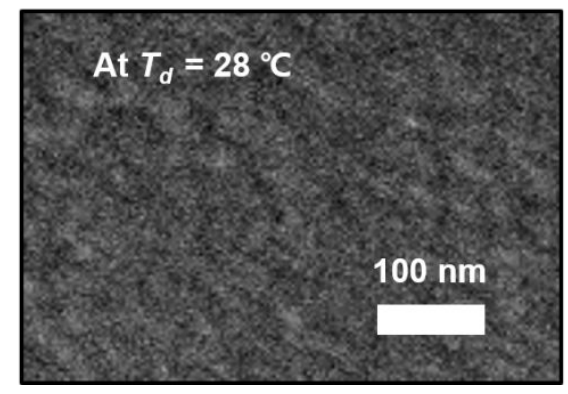

(b)

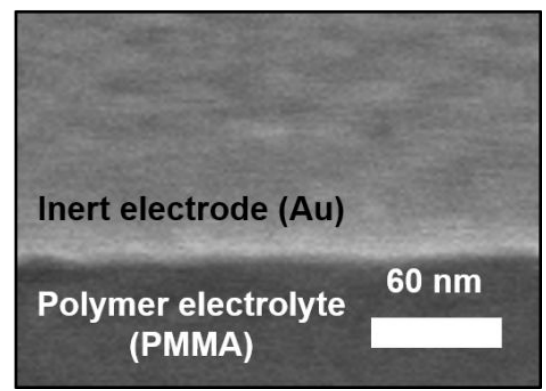

Figure S3. (a) The FE-SEM image of a $6 \mathrm{~nm}$-thick Au film deposited on the polymer electrolyte (PMMA) by thermal evaporation at $T_{\mathrm{d}}=28{ }^{\circ} \mathrm{C}$. (b) The cross sectional view of the Au/PMMA interface in the device without i-ITS.

(a)
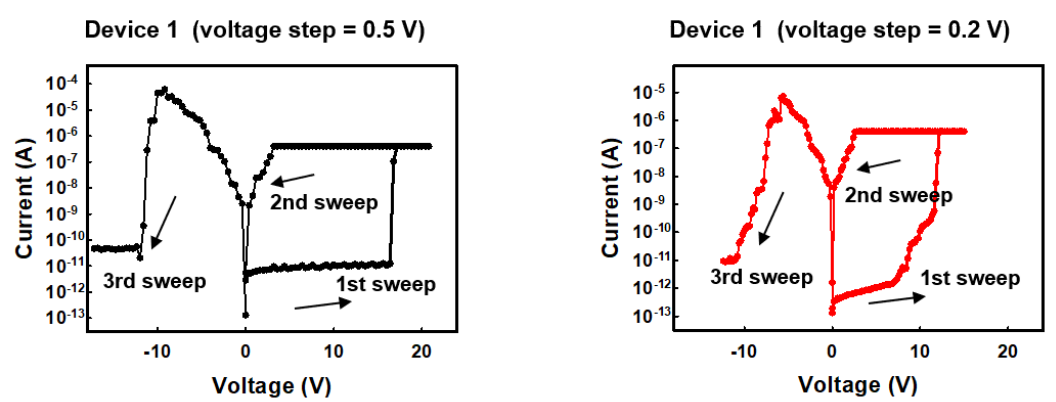

(b)
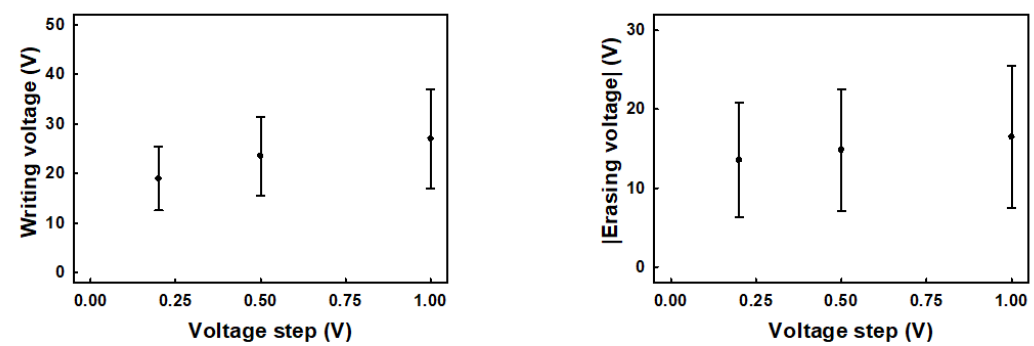

Figure S4. The effect of the voltage step on the electrical characteristics of the organic ECM device. (a) Current-voltage $(I-V)$ characteristics of Device 1 in the voltage-sweeping mode in steps of $0.5 \mathrm{~V}$ (left) and $0.2 \mathrm{~V}$ (right). (b) The operating voltages of Device 1, measured in three different voltage steps. 
(a)

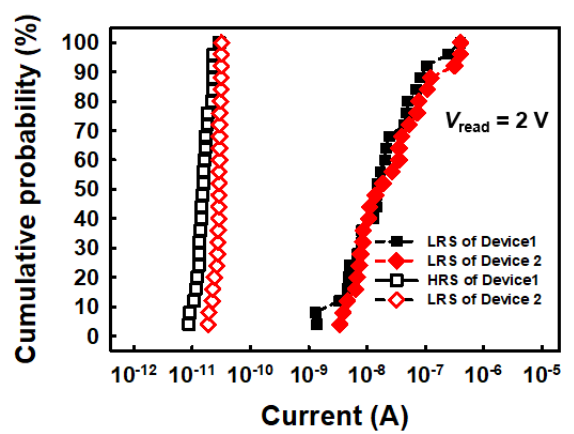

(b)

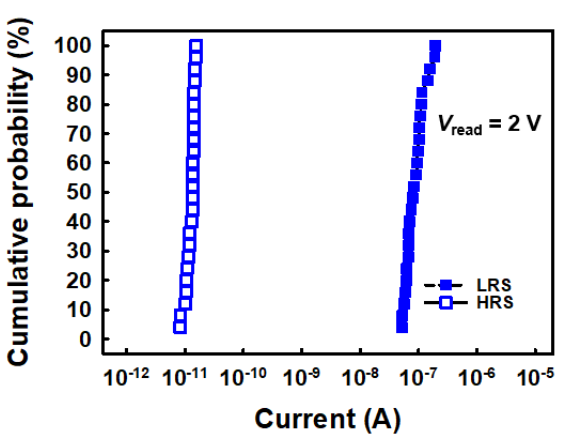

Figure S5. Statistical data of the current in the high resistance state (HRS) and that in the low resistance state (LRS) for (a) Device 1 without ITS, Device 2 with only a-ITSs, and (b) Device 3 with a-ITSs and iITSs. Each device was measured during 25 cycles.

(a)

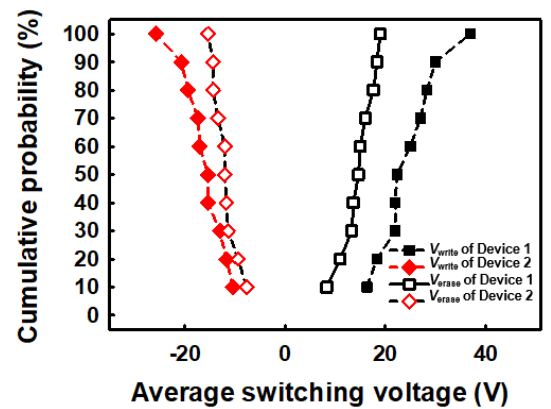

(b)

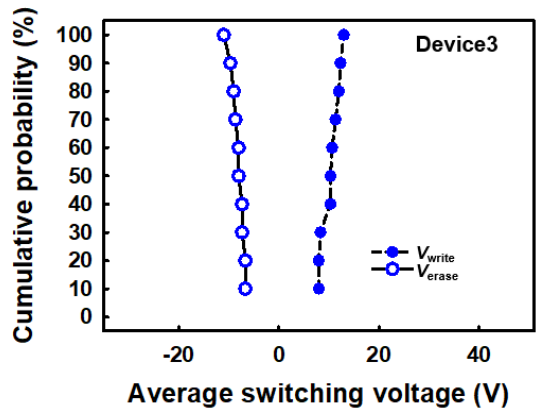

Figure S6. Statistical data of the average $V_{\text {write }}$ and $V_{\text {erase }}$ for (a) Device 1 without ITS, Device 2 with only a-ITSs, and (b) Device 3 with a-ITSs and i-ITSs. Total of 30 cells (10 for each type) were characterized and each cell was measured during 5 cycles for obtaining the average operating voltages. 
(a)

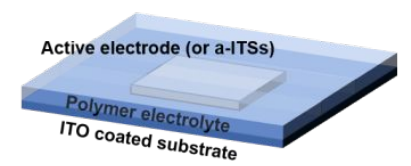

(b)

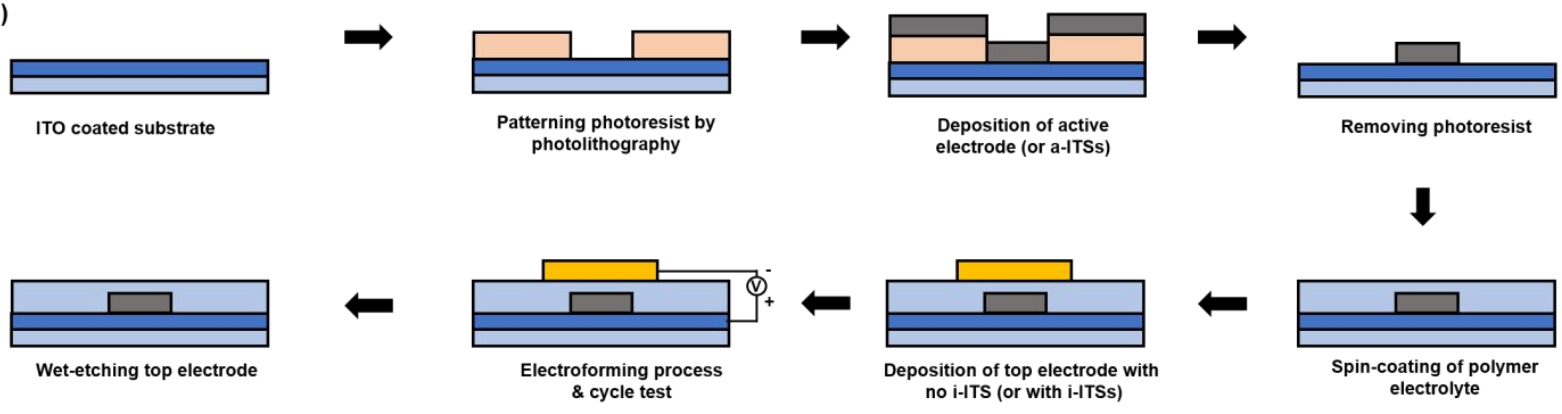

Figure S7. (a) A schematic diagram of a vertical-type organic ECM device fabricated for use in the C-AFM analysis. (b) A series of the steps for fabricating each vertical-type device. The physical size of all devices was $0.025 \times 0.025 \mathrm{~mm}^{2}$.

(a)

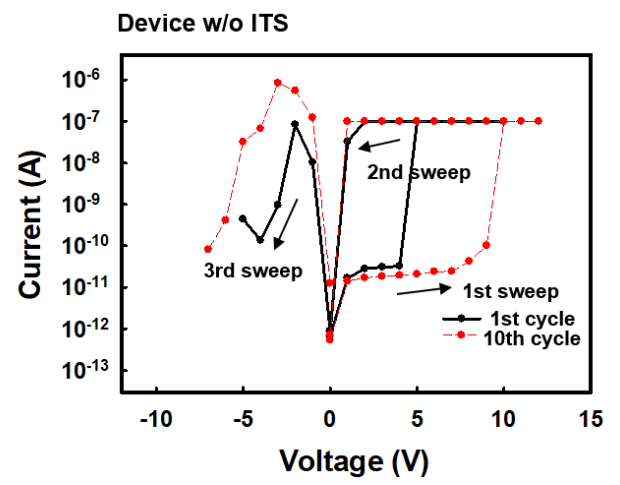

(b)

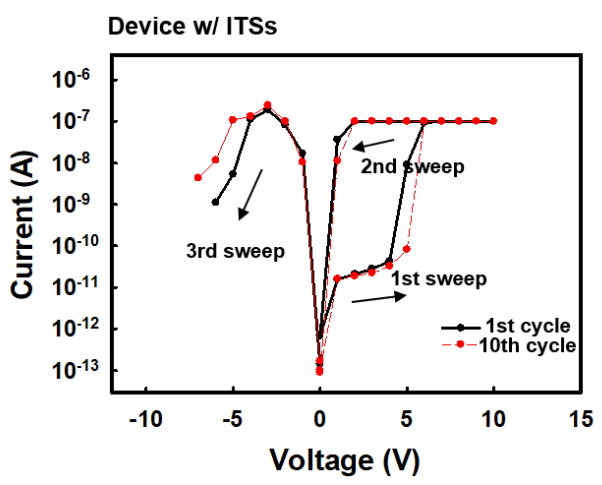

Figure S8. Current-voltage $(I-V)$ characteristics of the devices for the C-AFM analysis; (a) with no ITS and (b) with the ITSs. The values of $V_{\text {form }}$ are $37 \mathrm{~V}$ (with no ITS) and $12 \mathrm{~V}$ (with the ITSs) respectively. Note that all writing processes were performed at the compliance current of $1 \times 10^{-7} \mathrm{~A}$. 
(a)

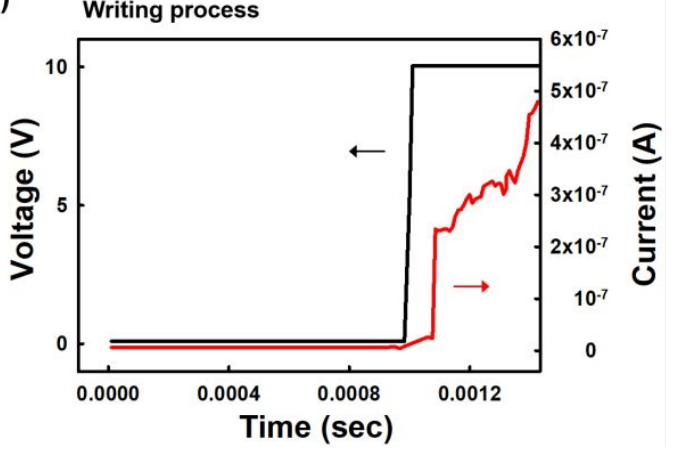

(b)

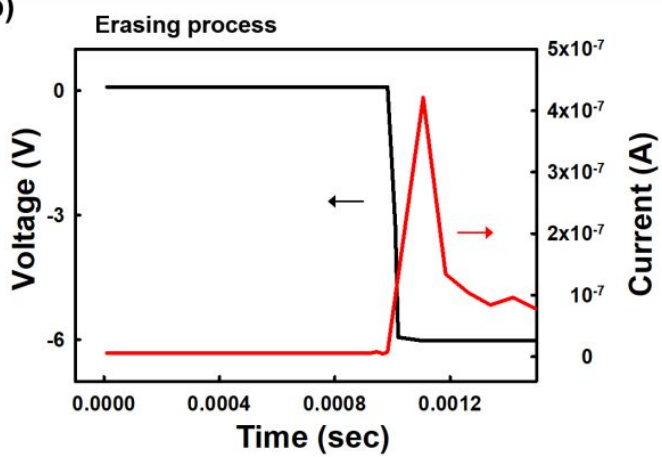

Figure S9. The transient responses of Device 3 to the switching electric pulses. (a) Voltage pulse with an amplitude of $10 \mathrm{~V}$ was used for writing and (b) the pulse with an amplitude $-6 \mathrm{~V}$ was used for erasing. The switching times for writing and erasing were about $70 \mu$ s and $150 \mu$ s respectively. 